\title{
Direct Numerical Simulations of Reaction Fronts Propagation under Quasi-Periodic Gravitational Modulation
}

\author{
Karam Allali \\ Department of Mathematics, University Hassan II- \\ Mohammedia \\ Po. Box 146, FST-Mohammadia, Morocco
}

\author{
Kamal El Karouni \\ Department of Mathematics, \\ University Hassan II-Mohammedia \\ Po. Box 146, FST-Mohammadia, Morocco
}

\begin{abstract}
The aim of this paper is to study the influence of quasiperiodic gravitational modulation on convective instability of reaction fronts in porous media. The model contains reaction diffusion equations coupled with the hydrodynamic equations under the Darcy-Boussinesq approximation. The direct numerical simulation of the dimensionless problem is fulfilled using the alternative direction method and the fast Fourier transform method. The convective instability boundary is found depending on the Lewis number and the amplitude of vibration.
\end{abstract}

\section{Keywords}

Direct numerical simulation, quasi-vibration, porous medium, reaction front.

\section{INTRODUCTION}

Propagation of reaction front behavior is intensively studied in relation with numerous physical, chemical, and biological problems; see for instance $[1,2,3,4,5,6,7]$. The influence of convective instability on reaction front propagation is studied in [8], liquid-liquid and liquid-solid cases are considered. In porous media, same study is carried out in [9], both of the reactant and the product are considered liquid. The influence of vibration on convective instability of reaction front propagation in porous media is investigated in [10], the convective instability boundary is found depending on the amplitude and the frequency of vibration.

Some works focused intention on the influence of quasiperiodic modulation on the convective instability of the boundary layer $[11,12,13]$. An asymptotic study is used to find the convective instability threshold depending on the frequencies ratio.

The linear stability analysis of the influence of quasi-periodic gravitational modulation on the convective instability in porous media taking in consideration zero inverse Lewis number, is studied in [14], the authors used two incommensurate frequencies and found the convective instability boundary depending on their ratio.

In this paper, we are interested in studying the influence of the quasi-periodic gravitational modulation on the convective instability of the reaction front in porous media. To this end, a direct numerical simulation of the dimensionless problem of the original one describing the phenomenon is investigated. The direct numerical simulation uses the alternate direction method for the reaction-diffusion equations and fast fourier transform for the hydrodynamic equations.

The paper is organized as follows. The problem is formulated in Section 2, followed in Section 3 by the direct numerical method. In Section 4, the result are presented and discussed. Section 5 concludes the work

\section{GOVERNING EQUATIONS}

\subsection{The model}

To study the influence of quasi-periodic vibration on the convective instability in porous media, we assume that the reaction front propagates in the opposite sense of gravity. The fluids acting in the reaction are assumed incompressible. A such phenomenon can be modeled by a system of reactiondiffusion equations coupled with the hydrodynamic equation under Darcy-Boussinesq approximation. In order to study the quasi-periodic effects, the reaction is submitted to quasiperiodic oscillations in the vertical direction. Therefore the fluid is under effect of gravitational acceleration $\mathrm{g}$ and a quasi-periodic oscillations $\lambda_{1} \sin \left(v_{1} t\right)+\lambda_{2} \sin \left(v_{2} t\right)$. Here $\lambda_{1}$ and $\lambda_{2}$ are the amplitudes, $v_{1}$ and $v_{2}$ are two incommensurate frequencies. The following equations are obtained :

$$
\begin{aligned}
& \frac{\partial T}{\partial t}+v \cdot \nabla T=\kappa \Delta T+q K(T) \emptyset(\alpha) \\
& \frac{\partial \alpha}{\partial t}+v \cdot \nabla \alpha=d \Delta \alpha+K(T) \emptyset(\alpha) \\
& v+\frac{K}{\mu} \nabla P=\frac{g \beta K}{\mu} \rho\left(T-T_{0}\right)\left(1+\left(\lambda_{1} \sin \left(v_{1} t\right)\right.\right. \\
& \left.\left.+\lambda_{2} \sin \left(v_{2} t\right)\right)\right) \gamma \\
& \nabla \cdot v=0
\end{aligned}
$$

With the following conditions:

$$
\begin{aligned}
& T=T_{i}, \alpha=1, \text { and } v=O \text { when } y \rightarrow+\infty \\
& T=T_{b}, \alpha=0, \text { and } v=O \text { when } y \rightarrow-\infty
\end{aligned}
$$

Here $\mathrm{T}$ is the temperature, $\alpha$ is the depth of conversion, $\mathrm{v}=\left(\mathrm{v}_{\mathrm{x}}, \mathrm{v}_{\mathrm{y}}\right)$, the fluid velocity, $\mathrm{P}$ the pressure, $\kappa$ the coefficient of thermal diffusivity, $\mathrm{d}$ the diffusion, $\mathrm{q}$ the adiabatic heat release, $g$ the gravity acceleration, $\rho$ the density, $\beta$ denotes the coefficient of thermal expansion, $\mu$ the viscosity 
and $\gamma$ the unit vector in the upward direction. In addition, T0 is the mean value of temperature, $\mathrm{Ti}$ is an initial temperature while $\mathrm{Tb}$ is the temperature of the burned mixture given by $T_{b}=T_{i}+q$

The function $K(T) \emptyset(\alpha)$ is the reaction rate where the temperature dependence is given by the Arrhenius exponent:

$K(T)=k_{0} \exp \left(-\frac{E}{R_{0} T}\right)$

$E$ is the activation energy, $R_{0}$ the universal gas constant and $\mathrm{k}_{0}$ the pre-exponential factor. For these direct numerical simulations, we will consider first order reaction, that is: $\phi(\alpha)=1-\alpha$.

\subsection{The dimensionless model}

In order to state the dimensionless model, the following space variables $x^{\prime}=\frac{x c_{1}}{\kappa}, y^{\prime}=\frac{y c_{1}}{\kappa}$, time $t^{\prime}=\frac{t c_{1}^{2}}{\kappa d}$, velocity $\frac{v}{c_{1}}$, pressure $\frac{P \kappa \mu}{K}$, with $c_{1}=c / \sqrt{2}$, frequencies $\sigma_{1}=\frac{\kappa}{c_{1}^{2}} v_{1}$ and $\sigma_{2}=\frac{\kappa}{c_{1}^{2}} v_{2}$ are introduced.

Denoting $\theta=\frac{T-T_{b}}{q}$ and keeping for convenience the same notation for the other variables, we obtain the following system

$$
\begin{aligned}
& \frac{\partial \theta}{\partial t}+v \cdot \nabla \theta=\Delta \theta+W_{z}(\theta) \phi(\alpha) \\
& \frac{\partial \alpha}{\partial t}+v \cdot \nabla \alpha=\Lambda \Delta \alpha+W_{z}(\theta) \phi(\alpha) \\
& \begin{array}{r}
v+\nabla P=R_{p}\left(\theta+\theta_{0}\right)\left(1+\lambda_{1} \sin \left(\sigma_{1} t\right)\right. \\
\left.+\lambda_{2} \sin \left(\sigma_{2} t\right)\right)\left(\begin{array}{l}
0 \\
1
\end{array}\right)
\end{array}
\end{aligned}
$$

$\nabla \cdot v=0$

With the following conditions:

$\theta=-1, \alpha=1$, and $v=O$ when $y \rightarrow+\infty$

$\theta=0, \alpha=0$, and $v=O$ when $y \rightarrow-\infty$

Here $\Lambda=d / \kappa$ denotes the inverse of the Lewis number, $R_{p}=\frac{K P_{r}^{2} R c_{1}^{2}}{\mu^{2}}$ where $R$ is the Rayleigh number and $P_{r}$ the Prandtl number defined by $R=\frac{g \beta q \kappa^{2}}{\mu C_{1}^{3}}$ and $P_{\mathrm{r}}=\frac{\mu}{\kappa}$. In addition, the parameters $\delta=\frac{R_{0} T_{b}}{E}$ and $\theta_{0}=\frac{T_{b}-T_{0}}{q}$ are used. The reaction rate is given by:

$W_{z}(\theta)=Z^{2} \exp \left(\frac{\theta}{Z^{-1}+\delta \theta}\right)$

where $Z=\frac{q E}{R_{0} T_{b}^{2}}$ stands for Zeldovich number.

\section{NUMERICAL METHOD}

In the case of large activation energy $E$, the small parameter $\delta$ in the reaction term formula (2.14) is neglected. Numerical computations are carried out using the stream function associated to the velocity. Due to incompressibility of the medium one can introduce the stream function $\psi$ defined by :

$$
v=\left(v_{x} ; v_{y}\right)=\left(\partial_{y} \psi ;-\partial_{x} \psi\right) .
$$

The system (2.8)-(2.13) is rewritten in the following form:

$\frac{\partial \theta}{\partial t}+\frac{\partial \psi}{\partial y} \frac{\partial \theta}{\partial x}-\frac{\partial \psi}{\partial x} \frac{\partial \theta}{\partial y}=\Delta \theta+z^{2} \exp (Z \theta)(1-\alpha)$

$$
\begin{aligned}
& \frac{\partial \alpha}{\partial t}+\frac{\partial \psi}{\partial y} \frac{\partial \alpha}{\partial x}-\frac{\partial \psi}{\partial x} \frac{\partial \alpha}{\partial y}=\Lambda \Delta \alpha+z^{2} \exp (Z \theta)(1-\alpha) 3.3 \\
& \Delta \psi=-R_{p} \frac{\partial \theta}{\partial x}\left(1+\lambda_{1} \sin \left(\sigma_{1} t\right)+\lambda_{2} \sin \left(\sigma_{2} t\right)\right)
\end{aligned}
$$

The conditions at infinity:

$\theta=-1, \alpha=1$ when $y \rightarrow+\infty$

$\theta=0, \alpha=0$, when $y \rightarrow-\infty$

This problem is studied numerically in a finite strip $\Omega=$ $\left(0 ; L_{x}\right) \times\left(0 ; L_{y}\right)$ with the noflux boundary conditions for the temperature and concentration and with the zero boundary condition for the function $\psi$. The initial condition is given by:

$\theta(0, x, y)=\theta_{0}(y), \alpha(0, x, y)=\alpha_{0}(y), \psi(0, x, y)=0$,

where the functions $\theta_{0}$ and $\alpha_{0}$ are some step functions. The system of equation (3.2), (3.3) is solved by using the alternating direction method. The descretization of our problem is given by the following:

$$
\begin{aligned}
\frac{\theta_{i, j}^{n+1 / 2}-\theta_{i, j}^{n}}{\delta_{t} / 2}+\frac{\psi_{i, j+1}^{n}-\psi_{i, j-1}^{n}}{2 \delta_{y}} \cdot \frac{\theta_{i+1, j}^{n+1 / 2}-\theta_{i-1, j}^{n+1 / 2}}{2 \delta_{x}} \\
-\frac{\psi_{i+1, j}^{n}-\psi_{i-1, j}^{n}}{2 \delta_{x}} \cdot \frac{\theta_{i, j+1}^{n}-\theta_{i, j-1}^{n}}{2 \delta_{y}} \\
=\frac{\theta_{i+1, j}^{n+1 / 2}-2 \theta_{i, j}^{n+1 / 2}+\theta_{i-1, j}^{n+1 / 2}}{\delta_{x}^{2}} \\
+\frac{\theta_{i, j+1}^{n}-2 \theta_{i, j}^{n}+\theta_{i, j-1}^{n}}{\delta_{y}^{2}} \\
+Z^{2} \exp \left(Z \theta_{i, j}^{n}\right)\left(1-\alpha_{i, j}^{n}\right)
\end{aligned}
$$

$$
\begin{aligned}
\frac{\theta_{i, j}^{n+1}-\theta_{i, j}^{n+1 / 2}}{\delta_{t} / 2}+ & \frac{\psi_{i, j+1}^{n}-\psi_{i, j-1}^{n}}{2 \delta_{y}} \cdot \frac{\theta_{i+1, j}^{n+1 / 2}-\theta_{i-1, j}^{n+1 / 2}}{2 \delta_{x}} \\
& -\frac{\psi_{i+1, j}^{n}-\psi_{i-1, j}^{n}}{2 \delta_{x}} \cdot \frac{\theta_{i, j+1}^{n}-\theta_{i, j-1}^{n}}{2 \delta_{y}} \\
& =\frac{\theta_{i+1, j}^{n+1 / 2}-2 \theta_{i, j}^{n+1 / 2}+\theta_{i-1, j}^{n+1 / 2}}{\delta_{x}^{2}} \\
& +\frac{\theta_{i, j+1}^{n+1}-2 \theta_{i, j}^{n+1}+\theta_{i, j-1}^{n+1}}{\delta_{y}^{2}} \\
& +Z^{2} \exp \left(Z \theta_{i, j}^{n}\right)\left(1-\alpha_{i, j}^{n}\right) \\
\frac{\alpha_{i, j}^{n+1 / 2}-\alpha_{i, j}^{n}}{\delta_{t} / 2}+\frac{\psi_{i, j}^{n}}{+1}-\psi_{i, j-1}^{n} \cdot \frac{\alpha_{i+1, j}^{n+1 / 2}-\alpha_{i-1, j}^{n+1 / 2}}{2 \delta_{x}} & -\frac{\psi_{i+1, j}^{n}-\psi_{i-1, j}^{n}}{2 \delta_{x}} \cdot \frac{\alpha_{i, j+1}^{n}-\alpha_{i, j-1}^{n}}{2 \delta_{y}} \\
& =\Lambda \frac{\alpha_{i+1, j}^{n+1 / 2}-2 \alpha_{i, j}^{n+1 / 2}+\alpha_{i-1, j}^{n+1 / 2}}{\delta_{x}^{2}} \\
& +\Lambda \frac{\alpha_{i, j+1}^{n}-2 \alpha_{i, j}^{n}+\alpha_{i, j-1}^{n}}{\delta_{y}^{2}} \\
& +Z^{2} \exp \left(Z \theta_{i, j}^{n}\right)\left(1-\alpha_{i, j}^{n}\right)
\end{aligned}
$$




$$
\begin{aligned}
\frac{\alpha_{i, j}^{n+1}-\alpha_{i, j}^{n+1 / 2}}{\delta_{t} / 2}+ & \frac{\psi_{i, j+1}^{n}-\psi_{i, j-1}^{n}}{2 \delta_{y}} \cdot \frac{\alpha_{i+1, j}^{n+1 / 2}-\alpha_{i-1, j}^{n+1 / 2}}{2 \delta_{x}} \\
& -\frac{\psi_{i+1, j}^{n}-\psi_{i-1, j}^{n}}{2 \delta_{x}} \cdot \frac{\alpha_{i, j+1}^{n}-\alpha_{i, j-1}^{n}}{2 \delta_{y}} \\
& =\Lambda \frac{\alpha_{i+1, j}^{n+1 / 2}-2 \alpha_{i, j}^{n+1 / 2}+\alpha_{i-1, j}^{n+1 / 2}}{\delta_{x}^{2}} \\
& +\Lambda \frac{\alpha_{i, j}^{n+1}-2 \alpha_{i, j}^{n+1}+\alpha_{i, j-1}^{n+1}}{\delta_{y}^{2}} \\
& +Z^{2} \exp \left(Z \theta_{i, j}^{n}\right)\left(1-\alpha_{i, j}^{n}\right)
\end{aligned}
$$

The fast Fourier transform method is used to solve the stream function equation (3.4).

\section{NUMERICAL RESULTS}

For the simulation we consider the following values of parameters: $Z=8, L_{x}=2, L_{y}=35, u=1.4142, \sigma_{1}=2500$, and $\sigma_{2}=\sqrt{2} \sigma_{1}$.

Figure 1 shows the critical Rayleigh number as function of the amplitude $\lambda_{1}$ for different values of $\lambda_{2}$ with $\Lambda=1$. It's observed that if the amplitude $\lambda_{2}$ increases, the front loses its stability. All the curves have the same asymptotic behavior for a large values of the amplitude $\lambda_{1}$. The stability of the reaction front is gained for a small values of the amplitudes $\lambda_{1}$ or $\lambda_{2}$.
Figure 2 shows variation of critical Rayleigh number as function of amplitude $\lambda_{1}$ with $\Lambda=0.1$ and for different values of amplitude $\lambda_{2}$. In this figure, some regions of reaction front stability are observed for small values of the amplitude $\lambda_{1}$. Figure 3 depicts the critical Rayleigh number as function of the amplitude $\lambda_{1}$, for a different values of the inverse of Lewis number $\Lambda$. it's shown that the front losses its stability when the inverse of Lewis number increases.

\section{CONCLUSION}

In this work, the effect of the quasi-periodic gravitational modulation on the convective instability of reaction front in porous media is investigated. The case of a heating from below is considered when the sense of reaction is opposite to the sense of gravity. The alternating direction method for the reaction differentials equations and the fast Fourier transform for the stream function equations are used.

The quasi-periodic vibrations produce a stabilizing or a destabilizing effect, which strongly depends on the amplitudes of vibrations and the inverse of Lewis number. For small amplitudes the front is stable, but when the amplitudes are increased significantly the front loses its stability and becomes less stable. In summary, the study considered showed that the amplitudes and the inverse of Lewis number play an important role in controlling the convective instability of reaction front.

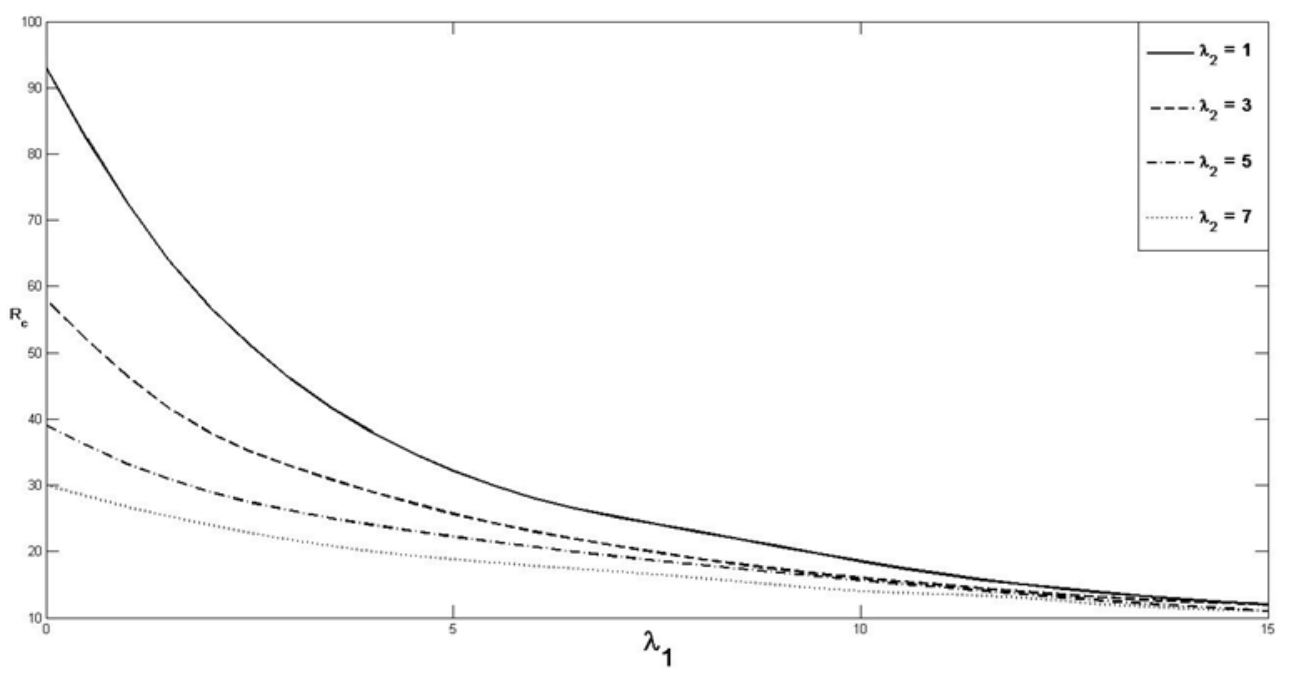

Fig 1: Critical Rayleigh number as function of the amplitude $\lambda_{1}$ for $\Lambda=1$ and different values of $\lambda_{2}$ 


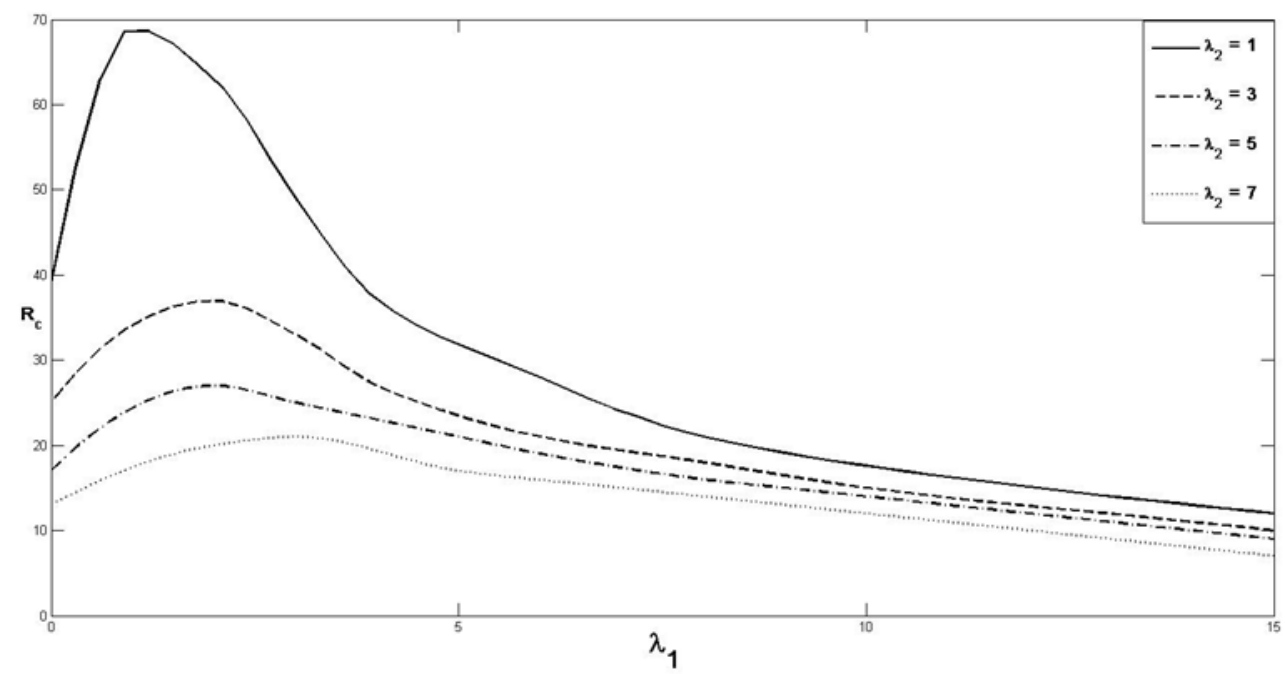

Fig 2: Critical Rayleigh number as function of the amplitude $\lambda_{1}$ for $\Lambda=0.1$ and different values of $\lambda_{2}$

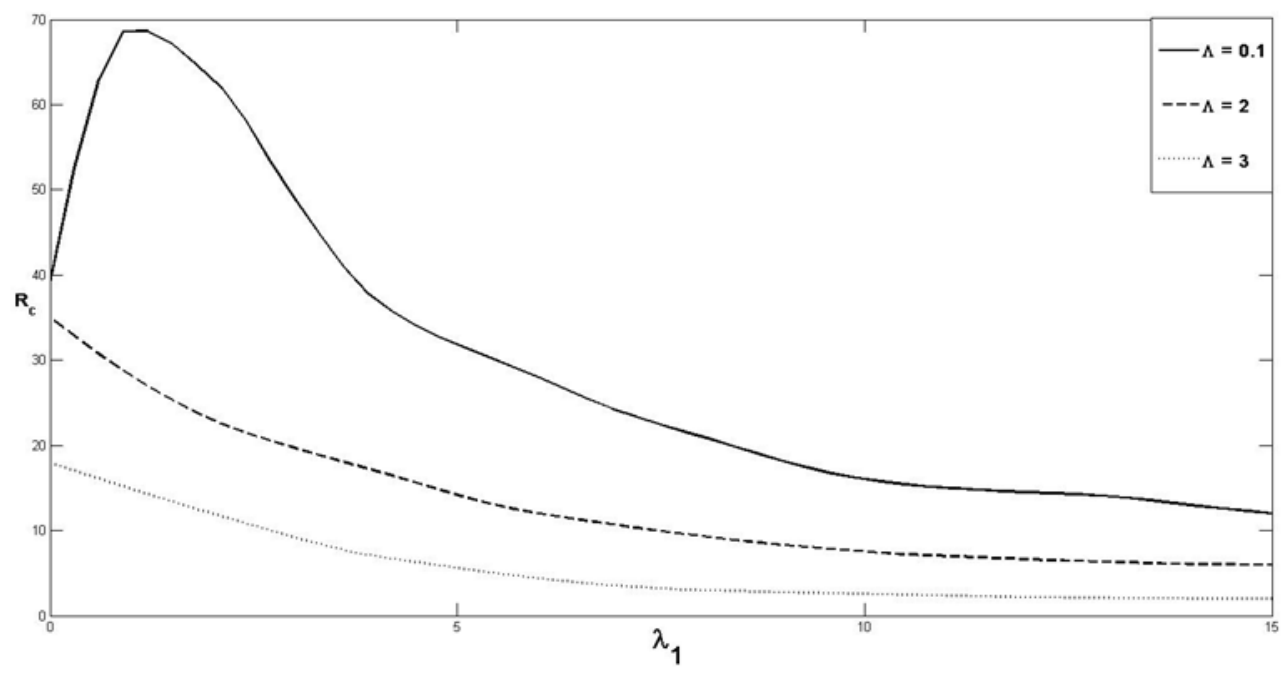

Fig 3: Critical Rayleigh number as function of the amplitude $\lambda_{1}$ for $\lambda_{2}=1$ and different values of $\Lambda$

\section{REFERENCES}

[1] Aronson, D., Weinberger, H., 1975. Nonlinear Diffusion in Population Genetics, Combustion and Nerve Propagation; Lecture Note in Math Vol, 446, pringerVerlag, Berlin, 1975.

[2] Brunet, E., Derrida, B., 1997. Shift in the velocity of a front due to a cutoff, Phys. Rev. E 56 (1997), 25972604.

[3] Rogers, J. L., Schatz, M. F., Bougie, J. L., Swift, J. B., 2000. Rayleigh-Bnard convection in a vertically oscillated fluid layer, Phys. Rev. Lett. 84 (2000), no. 1, 87-90.

[4] Murray, J.D., 1989. Mathematical Biology, SpringerVerlag, Berlin.
[5] Volpert, V., Petrovskii, S., 2009. Reaction-diffusion waves in biology, Physics of Life Reviews, 6 (2009), 267-310.

[6] Volpert, A., Volpert, Vit, Volpert, Vl.,1994. Traveling wave solutions of parabolic system, American Mathematical Society, Providence, RI, (1994) 448 pp.

[7] Freidlin, M., Markov, 1996. Processes and Differential Equations: Asymptotic Problems, Birkhauser, Basel.

[8] Garbey, M., Taik, A., Volpert V., 1998. Influence of naturel convection on stability of reaction fronts in liquids, Quart Appl Math 1998; 53,135.

[9] Allali, K., Ducrot, A., Taik, A., Volpert, V., 2007. Convective instability of reaction fronts in porous media, Math. Model. Nat. Phenom. 2 (2007), no. 2, 20-39. 
[10] Aatif, H., Allali, K., El Karouni, K., 2010. Influence of Vibrations on Convective Instability, Math. Model. Nat. Phenom. Vol. 5, No. 5, (2010) 123-137.

[11] Boulal, T., Aniss, S., Belhaq, M., Rand, R., 2007. Effect of quasiperiodic gravitational modulation on the stability of a heated fluid layer, Phys. Rev. E. 52 (2007), 76,56320 .

[12] Boulal, T., Aniss, S., Belhaq, M., Azouan, A., 2008. Effect of quasi-periodic gravitational modulation on the convective instability in Hele-Shaw cell. International Journal of Non-Linear Mechanics 2008; 43:852-7.
[13] Boulal, T., Aniss, A., Belhaq, M., 2008. Quasiperiodic gravitational modulation of convection in magneticfluid, Thermal Non-Equilibrium, Lecture Notes of the 8th International meeting of thermodiffusion, 9-13 June, 2008, Bonn, Germany, edited by S. Wiegand, W. K"ohler, J.K.G, Dhont (2008), (300) pages ISBN: 978-389336-523-4.

[14] Allali, K., Belhaq, M., El Karouni, K., 2012. Influence of quasi-periodic gravitational modulation on convective instability of reaction fronts in porous media, Commun Nolinear Sci Numer Simulat 17(2012)1588-1596. 